\title{
Complete Sequence Confirmation of Large Peptides by High Energy Collisional Activation of Multiply Protonated Ions
}

\author{
V. S. Kumar Kolli and Ron Orlando \\ Complex Carbohydrate Research Center and Department of Biochemistry and Molecular Biology, \\ University of Georgia, Athens, Georgia, USA
}

\begin{abstract}
High energy collisional activation on a tandem four-sector mass spectrometer produces fragmentation throughout the peptide backbone of multiply protonated peptides in the 4-5-ku molecular weight range, which enables complete sequence confirmation from these peptides, including leucine-isoleucine determinations. The resolutions provided by this type of mass spectrometer permit the charge state of the fragment ions to be determined by identifying their isotopic spacing. This eliminates the complexity in spectral interpretation caused by the presence of fragment ions in various charge states. The reliable mass assignments from a sector mass spectrometer permit identification of amino acids that differ by as little as a single unit in these large peptides. The combination of excellent mass accuracy with the ability to assign charge states is expected to greatly facilitate the interpretation of tandem mass spectra from unknown peptides, and permit their sequences to be deduced. These results lead us to propose tandem mass spectrometric analysis of multiply charged precursor ions on a tandem four-sector instrument as a direct strategy for determination of the complete amino acid sequence of peptides up to $5 \mathrm{ku}$. (J Am Soc Mass Spectrom $1995,6,234-241)$
\end{abstract}

$\mathrm{T}$ landem mass spectrometry offers several advantages over traditional Edman degradation in the sequence analysis of proteins: the abilities to sequence peptides present in mixtures [1,2], identify modified amino acids $[1,3]$, sequence peptides with blocked $N$-termini $[1,4]$, and significantly reduce sample consumption [5]. In tandem mass spectrometry the sample is ionized and the first mass spectrometer provides separation based upon the mass-to-charge ratio of the primary ions. An ion at a single mass-to-charge ratio value is then selected and passed into a collision chamber between the two mass spectrometers where it is fragmented by collision with an inert target gas, that is, collisional activation (CA). The fragment ions are then detected as they pass through the second mass spectrometer where they are analyzed based upon their mass-to-charge ratio values.

Currently, tandem mass spectrometry sequencing is limited by the mass range of peptides amenable to this process, that is, peptides with molecular weights up to $3.5 \mathrm{ku}[6]$. A possible explanation for this upper mass limit is the inverse relationship between the size of the precursor ion and the maximum amount of energy deposited during the collision process. Similarly, the

Address reprint requests to Ron Orlando, Complex Carbohydrate Research Center and Department of Biochemistry, University of Georgia, 220 Riverbend Road, Athens, GA 30602-4712. increased number of degrees of freedom also may limit the size of peptide that can be sequenced by tandem mass spectrometry because of the decreased dissociation rate with increased precursor ion size.

Very large $(66-\mathrm{ku})$ multiply charged ions have been fragmented by low energy CA [7-10]. These ions appear to fragment more easily than their singly charged counterparts, presumably due to intramolecular charge repulsion. However, interpretation of these CA spectra is hampered by the presence of a large number of variously charged ions. Also, only limited sequence information is obtained from these experiments because fragment ions appear from limited regions of the analyte ion [7-10]. Therefore, although large proteins have been fragmented successfully, the majority of the peptides that have either been sequenced or had their sequences confirmed by electrospray ionization (ESI) tandem mass spectrometry lie within the mass range allowed by high energy CA of singly charged precursor ions.

We have demonstrated that high energy CA of large doubly charged peptide ions produces backbone and combined backbone-side-chain fragmentations identical to those observed for singly charged peptide ions [11-14]. Other laboratories have observed similar behavior with high energy CA of peptide ions in higher charge states $[15,16]$. In addition, we have shown that these fragment ions occur throughout the precursor 
and permit virtually complete sequence confirmation for peptides over $5 \mathrm{ku}[13,14]$.

Our previous studies, unfortunately, required extremely large sample quantities $(10-100 \mathrm{nmol})$ that made this scheme impractical for biological problemsolving. This need for large sample quantities presumably resulted from the inefficient production of doubly charged ions by fast-atom bombardment (FAB), the ionization technique used in our earlier work. This ionization process also limited those studies to the analysis of doubly charged precursor ions, which led to difficulty in the determination of the amino acids located in the exact center of the peptide. In these reports, we predicted that the use of ESI as the ionization technique would significantly reduce sample requirements, because ESI appears to produce more abundant multiply charged ions than FAB. In addition, the presence of ions in various charge states was expected to allow us to identify the amino acids in the middle of the peptide. We stated that we expected the higher resolutions provided by four-sector mass spectrometers to permit charge state determinations of the fragment ions and aid a priori sequence determinations. These predictions led us to undertake this study on the use of ESI tandem mass spectrometry on a four-sector tandem mass spectrometer for sequence confirmation of peptides over $4 \mathrm{ku}$.

\section{Experimental}

All experiments were performed on a JEOL (Tokyo, Japan) $\mathrm{HX} / \mathrm{HX} 110 \mathrm{~A}$ tandem four-sector mass spectrometer. Precursor ions were generated by a JEOL electrospray ionization source (JEOL MS-ESI), operated at an accelerating voltage of $7 \mathrm{kV}$. All experiments were performed on positive ions with a resolution of approximately 500 ( $90 \%$ valley) in MS1. MS2 had an approximate resolution of 3600 (50\% valley) for all experiments, except for the tandem mass spectrum of human Tyr-corticotropin releasing factor in which a resolution of approximately 2100 (50\% valley) was used. Collisional activation was performed in the third field-free region by using helium as the collision gas at a pressure sufficient to attenuate the primary ion beam by $75 \%$. The collision cell was floated at $6 \mathrm{kV}$, that is, 1 $\mathrm{kV}$ below the acceleration voltage. These conditions provide a laboratory collision energy equal to $1 \mathrm{kV} \times$ (the charge state of the precursor ion). We have reported a detailed derivation of the linked scan that was used here to detect the CA products of multiply charged ions in a floating collision cell [11, 12]. Fragment ions were detected by a JEOL MS-ADS11 variable dispersion array detector [17]. These spectra were recorded with a fragment ion dispersion of 1 to 1.1 (10\%). A delay time of $0.1 \mathrm{~s}$ was used for stepping the magnetic and electric sectors to allow stabilization of the magnetic field.

All samples were acquired from Sigma Chemical Co. (St. Louis, MO) and were used without further purification. All peptides except for human Tyr-corticotropin releasing factor were dissolved in a 49:49:2 mixture of water-methanol-glacial acetic acid to a concentration of $50 \mathrm{pmol} / \mu \mathrm{L}$. Human Tyr-corticotropin releasing factor was dissolved in a 49:49:2 mixture of water-methanol-glacial acetic acid to a concentration of $1 \mathrm{pmol} / \mu \mathrm{L}$. These solutions were introduced to the ESI source at a flow rate of $1 \mu \mathrm{L} / \mathrm{min}$. Each sample required approximately $10 \mathrm{~min}$ to tune the instrument, set up the experimental parameters, and permit acquisition of the mass and tandem mass spectra. Consequently, approximately $10-500$ pmol of sample is required for this approach.

\section{Results and Discussion}

Pancreatic polypeptide, human, $M W=4181.7 \mathrm{u}$. The $[\mathrm{M}+5 \mathrm{H}]^{5+}$ ion, the most abundant species observed in the ESI spectrum of this peptide, was selected for tandem mass spectrometric analysis (Figure 1). Low mass-to-charge ratio immonium ions identify the presence of $I / L, D, Q, M, R$, and $Y$ residues in the sequence. The majority of sequence-specific fragment ions contain the original $C$-terminus as expected by the high concentration of basic amino acids at this terminus (see the fragmentation scheme for this polypeptide in Figure 2). Fragmentation occurs throughout the peptide, and the resulting fragment ions verify the sequence of all amino acids except $\mathrm{I}_{28}$ and $\mathrm{N}_{29}$. Several fragment ions that contain the original $N$-terminus confirm the amino acid sequence from $R_{26}$ to the $C$-terminus. The two series of fragment ions provided complementary information and permitted complete sequence confirmation of this peptide.

The observed fragment ions and their charge states can be rationalized based upon the most likely protonation sites, that is, the positions of the most basic amino acid residues. In this peptide, these protonation sites appear to be the four arginine residues located at positions $25,26,33$, and 35 . The C-terminus fragment ions that possess all of the arginine residues appear as quadruply charged ions, whereas fragments that contain three, two, and one arginine residue(s) appear as triply, doubly, and singly charged ions, respectively. Hence, the charge state of the C-terminus fragment ions equals the number of arginine residues present in the fragment, which implies that each of the arginine residues possesses an extra proton. This result was surprising, because two arginines (i.e., protonation sites) are adjacent and we had initially postulated that the coulombic repulsion between two charges on adjacent amino acids would preclude protonation of both sites. These observations are in direct contrast to a previous report on tandem mass spectrometric analysis of multiply charged peptides that suggested that the excess protons were spread across the peptide [15]. However, it is clear from the observed charge state of the C-terminus fragment ions that both of these adjacent arginine residues are protonated, that is, frag- 

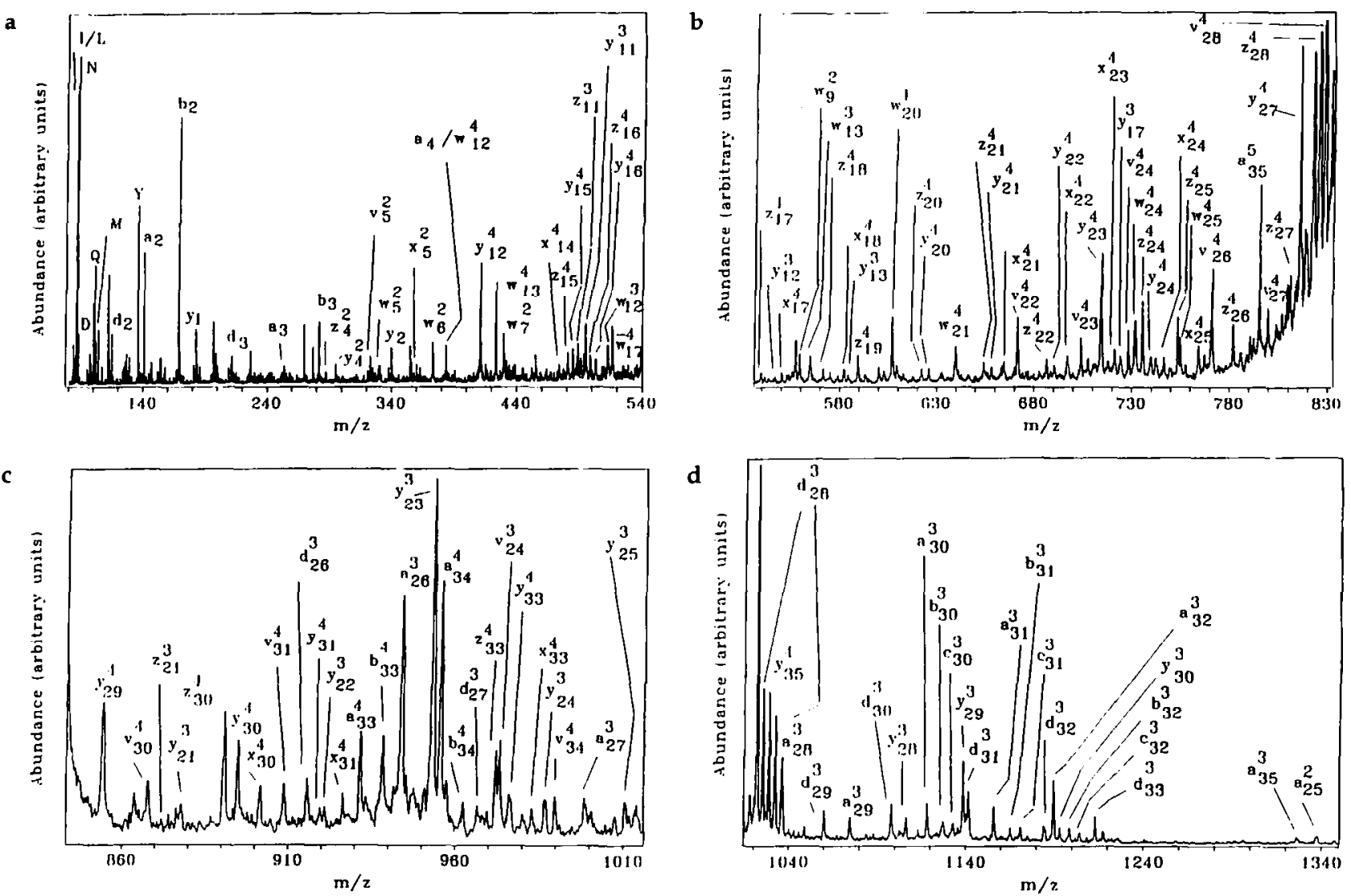

Figure 1. Product ion spectrum of the quintuply protonated molecular ion $[\mathrm{M}+5 \mathrm{H}]^{5+}$ of pancreatic polypeptide (human), $\mathrm{MW}=4181.7 \mathrm{u}$.

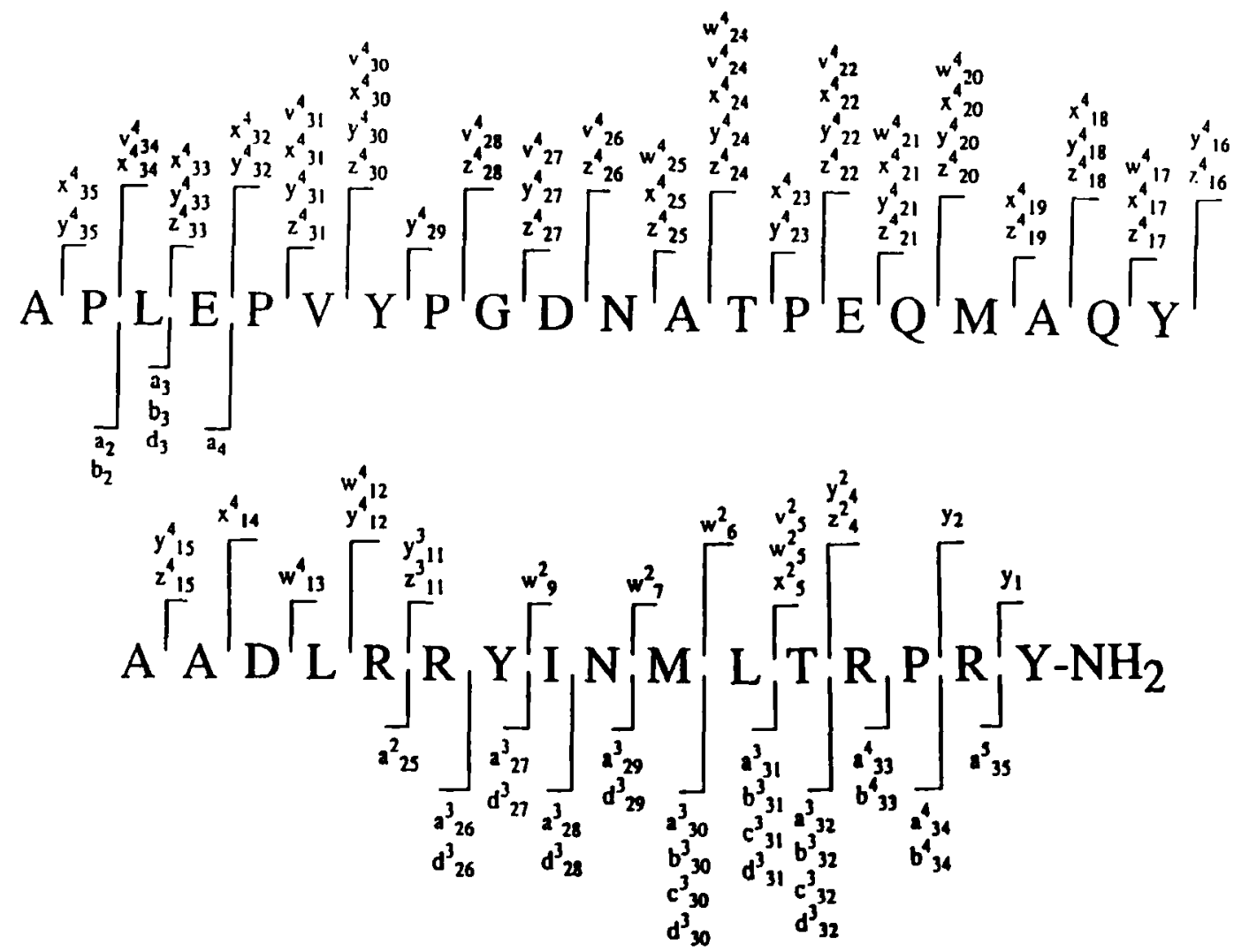

Figure 2. Fragment ions observed upon $C A$ of the quintuply protonated molecular ion $[\mathrm{M}+5 \mathrm{H}]^{5+}$ of pancreatic polypeptide (human), MW $=4181.7 \mathrm{u}$. 
ments that do not contain either $\mathrm{R}_{25}$ or $\mathrm{R}_{26}$ appear as doubly charged, fragment ions that result from cleavage between the two arginines $\left(y_{11}\right.$ and $\left.z_{11}\right)$ appear as triply charged, and fragments that possess both of these arginines (in particular $x_{12}$ and $y_{12}$ ) are quadruply charged. These charge states unambiguously established that the proton affinity of the arginine residues more than offsets the electrostatic repulsion of two protons on adjacent residues. In the case of the $N$ terminus fragments, the charge state is 1 greater than the number of arginine residues present, which implies that a proton is also localized at the $\mathrm{N}$-terminus amino group in a certain population of precursor ions. The charge states of the $N$-terminus fragment ions also confirm that adjacent arginine residues are protonated.

It should be noted that ions were not observed above the mass-to-charge ratio region shown in Figure 1 , even though the second mass spectrometer was scanned well above the mass-to-charge ratio of the singly charged species. This was also the case for the other peptides analyzed in this study. These results initially appear to contradict one of our previous studies on high energy CA of doubly protonated peptides, in which we reported transfer of an electron from the collision gas to the precursor ion [14]. We attribute this difference to the use of xenon as the collision gas in our earlier work and helium in the current study, because the probability of electron transfer from xenon to the sample ion is greater because this gas has a lower ionization potential than helium.

Similar results were obtained from the $[\mathrm{M}+5 \mathrm{H}]^{5+}$ ion of bovine pancreatic polypeptide, $\mathrm{MW}=4225.8 \mathrm{u}$, and the $[\mathrm{M}+3 \mathrm{H}]^{3+}$ ion of avian pancreatic polypeptide, $\mathrm{MW}=4237.6 \mathrm{u}$ (data not shown), as expected from the high sequence homology of these polypeptides. These ESI tandem mass spectra permitted virtually complete sequence confirmation, and the charge states of the detected fragment ions generally agreed with the number of basic amino acids in the fragment.

Galanin message-associated peptide, fragment 1-41 amide, porcine, $M W=4643.2 u$. The $[\mathrm{M}+5 \mathrm{H}]^{5+}$ ion is the most abundant species observed in the ESI spectrum of this peptide and was selected for tandem mass spectrometric analysis (Figure 3 ). Low mass-to-charge ratio immonium ions identify the presence of $I / L, D, H$, and $\mathrm{K}$ residues in the sequence. This peptide contains six basic residues distributed fairly evenly throughout the sequence, that is, arginines at positions 9, 15, and 24, lysines at positions 21 and 36, and histidine at position 34. This distribution of basic amino acid residues accounts for the distribution of both $\mathrm{N}$ - and C-terminus sequence-specific fragment ions, which confirms the majority of this peptide sequence (see the fragmentation scheme for this peptide in Figure 4). Once again, the charge states of the observed fragment ions correlate well with the number of basic amino acids contained in the fragment ion.
Tyr-corticotropin releasing factor, human, $M W=4920.7 u$. The $[\mathrm{M}+6 \mathrm{H}]^{6+}$ ion is the most abundant species observed in the ESI spectrum of this peptide and was selected for tandem mass spectrometric analysis (Figure 5). Low mass-to-charge ratio immonium ions identify the presence of $I / L, Q, E, H$, and $Y$ residues in the sequence. This peptide contains six basic residues distributed fairly evenly throughout the sequence, that is, arginines at positions 17,24 , and 36 , lysines at position 37, and histidines at positions 14 and 33. This distribution of basic amino acid residues accounts for the distribution of both $\mathrm{N}$ - and C-terminus sequencespecific fragment ions, which confirms the entire sequence of this peptide (see the fragmentation scheme for this peptide in Figure 6). Once again, the charge states of the observed fragment ions correlate well with the number of basic amino acids contained in the fragment ion.

Comparison of tandem mass spectra from different charge states. Figure 7 shows a comparison of the low mass regions of the tandem mass spectra from the triply and the quadruply protonated molecular ions of the avian pancreatic polypeptide. These regions from these two spectra are essentially identical, that is, they possess the same fragment ions in approximately the same abundance with the same charge states. In addition, the higher mass regions of these two spectra (not
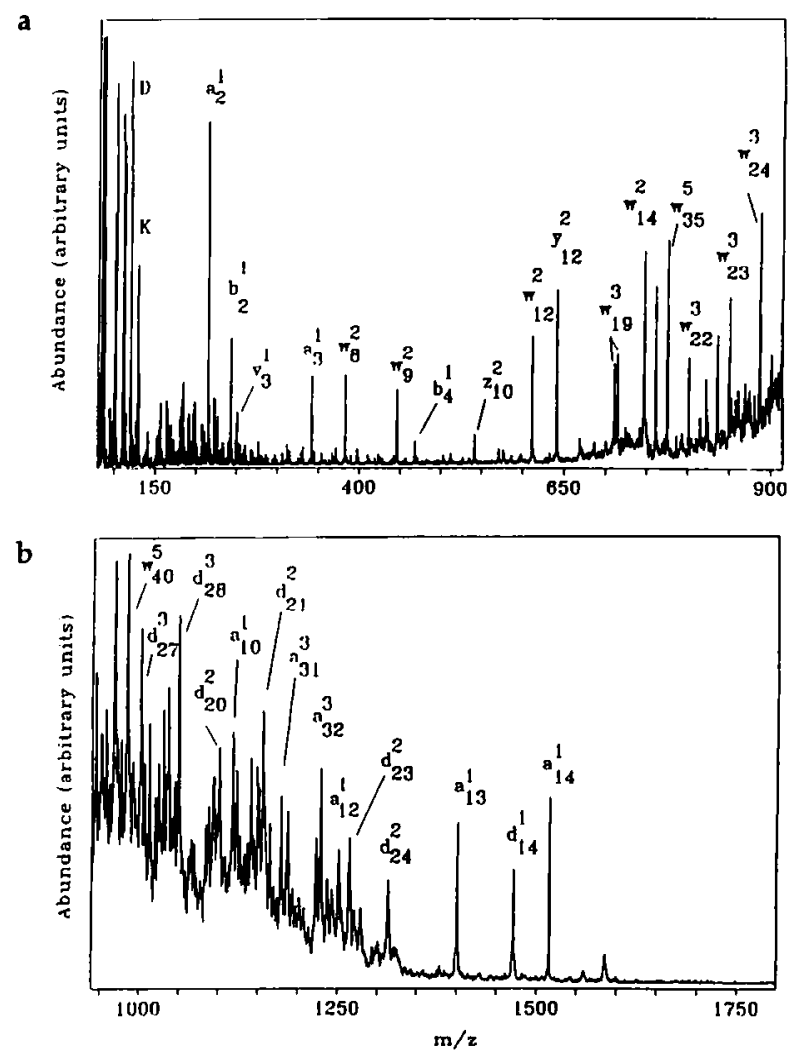

Figure 3. Product ion spectrum of the quintuply protonated molecular ion $[\mathrm{M}+5 \mathrm{H}]^{5+}$ of galanin message-associated peptide fragment 1-41 amide (porcine), $\mathrm{MW}=4643.2 \mathrm{u}$. 

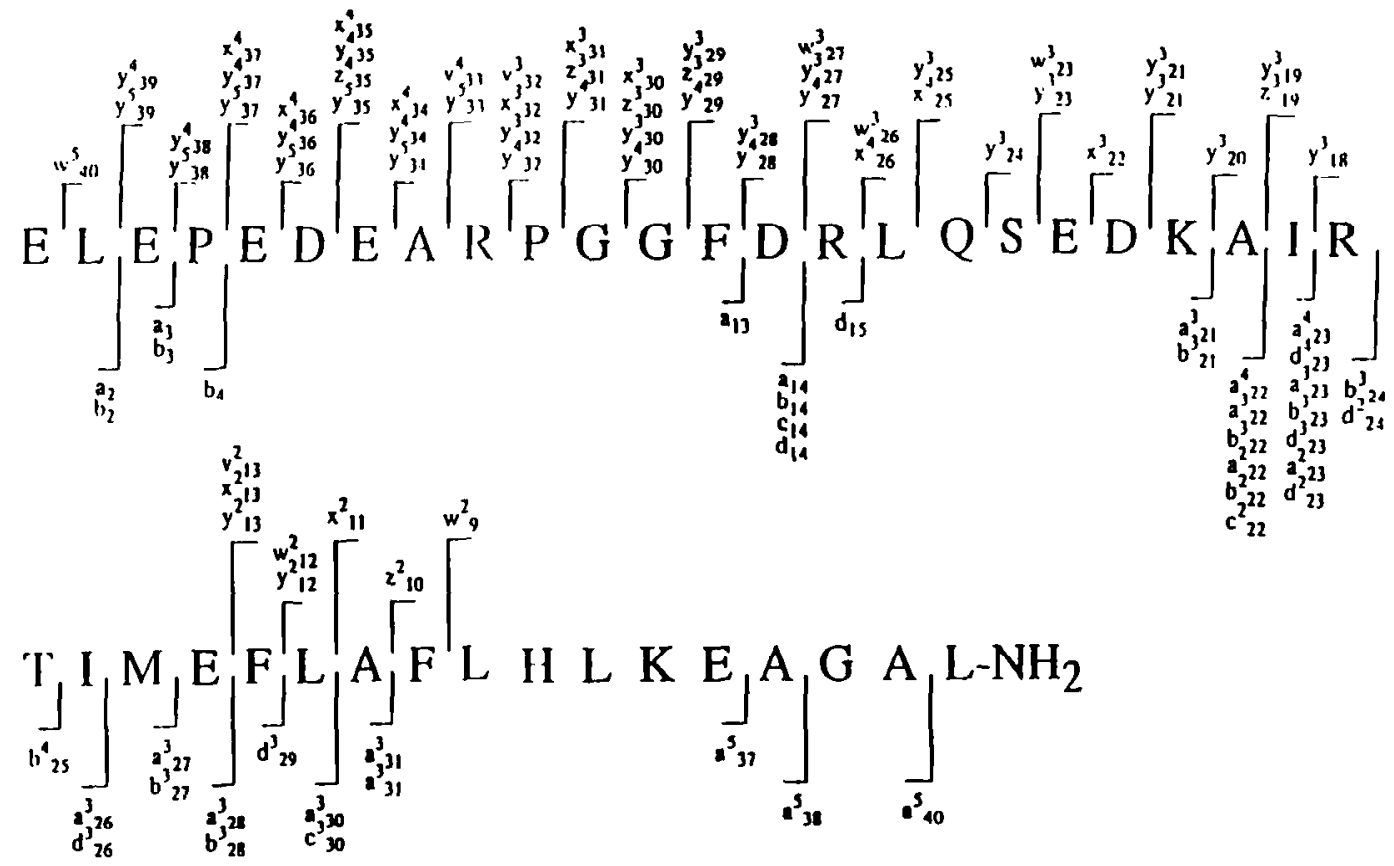

Figure 4. Fragment ions observed upon $C A$ of the quintuply protonated molecular ion $[\mathrm{M}+5 \mathrm{H}]^{5+}$ of galanin message-associated peptide fragment $1-41$ amide (porcine), $\mathrm{MW}=4643.2 \mathrm{u}$.
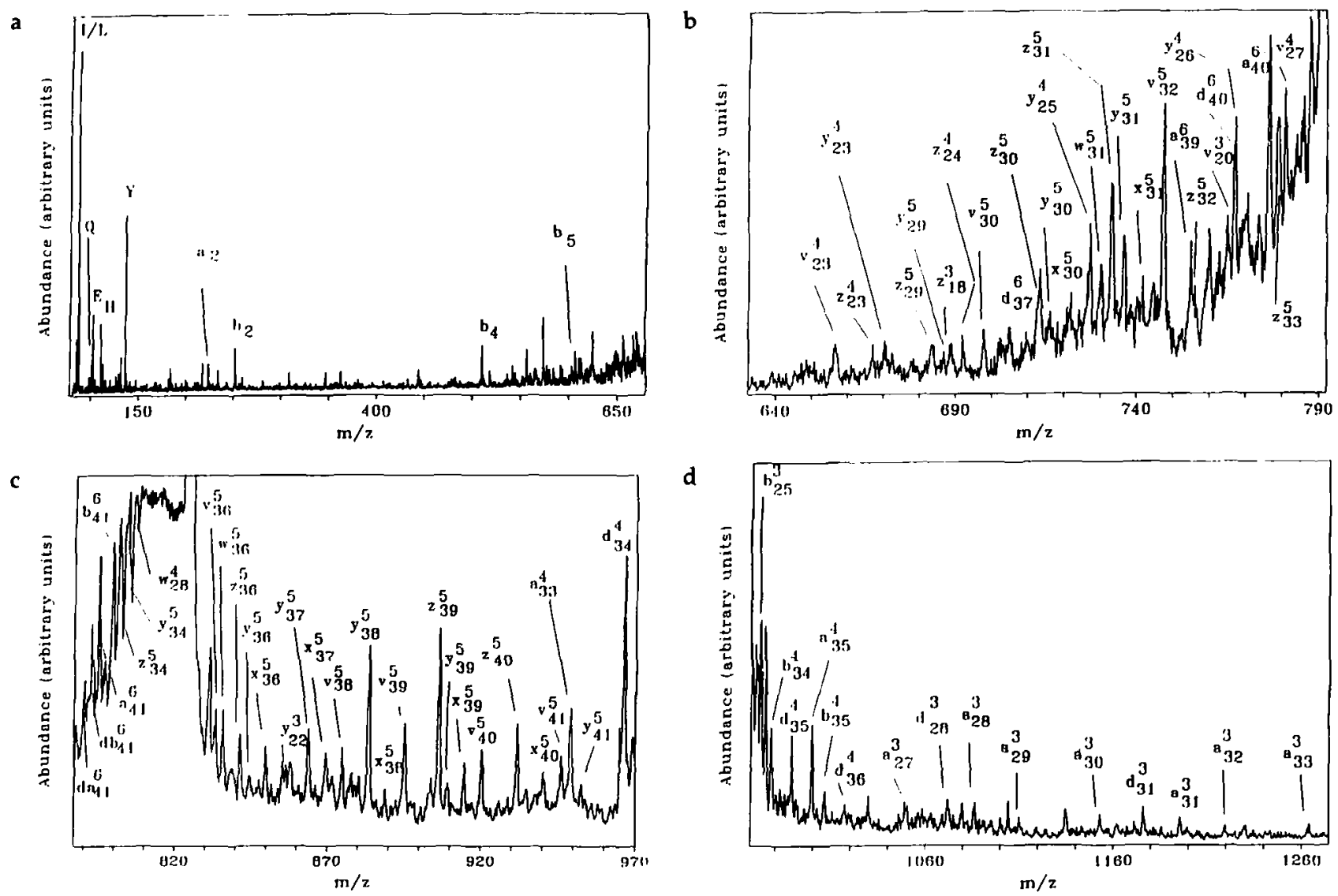

Figure 5. Product ion spectrum of the sextuply protonated molecular ion $[\mathrm{M}+6 \mathrm{H}]^{6+}$ of Tyr-corticotropin releasing factor (human), $\mathrm{MW}=4920.7 \mathrm{u}$. 


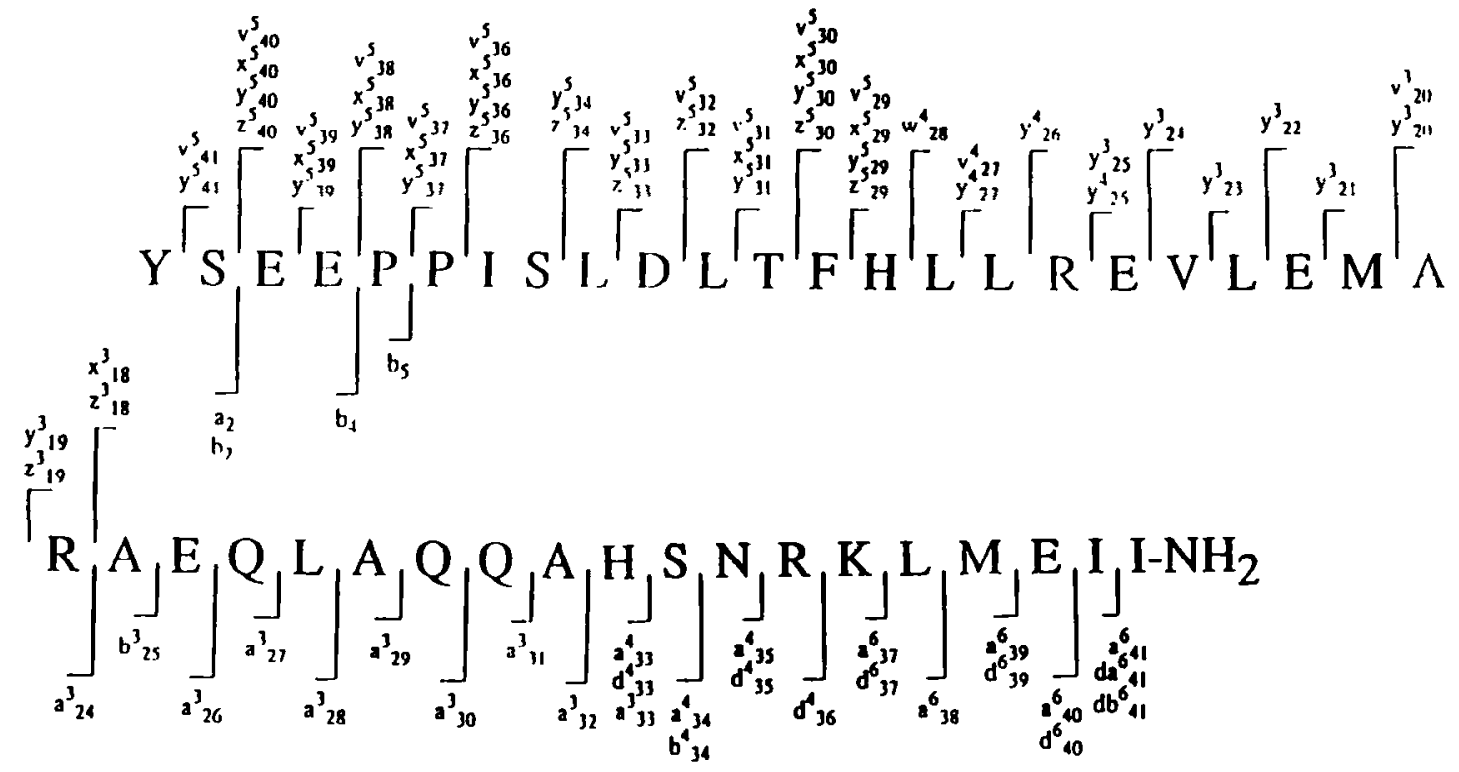

Figure 6. Fragment ions observed upon CA of the sextuply protonated molecular ion $[\mathrm{M}+6 \mathrm{H}]^{6+}$ of human recombinant Tyr-corticotropin releasing factor (human), $\mathrm{MW}=4920.7 \mathrm{u}$.

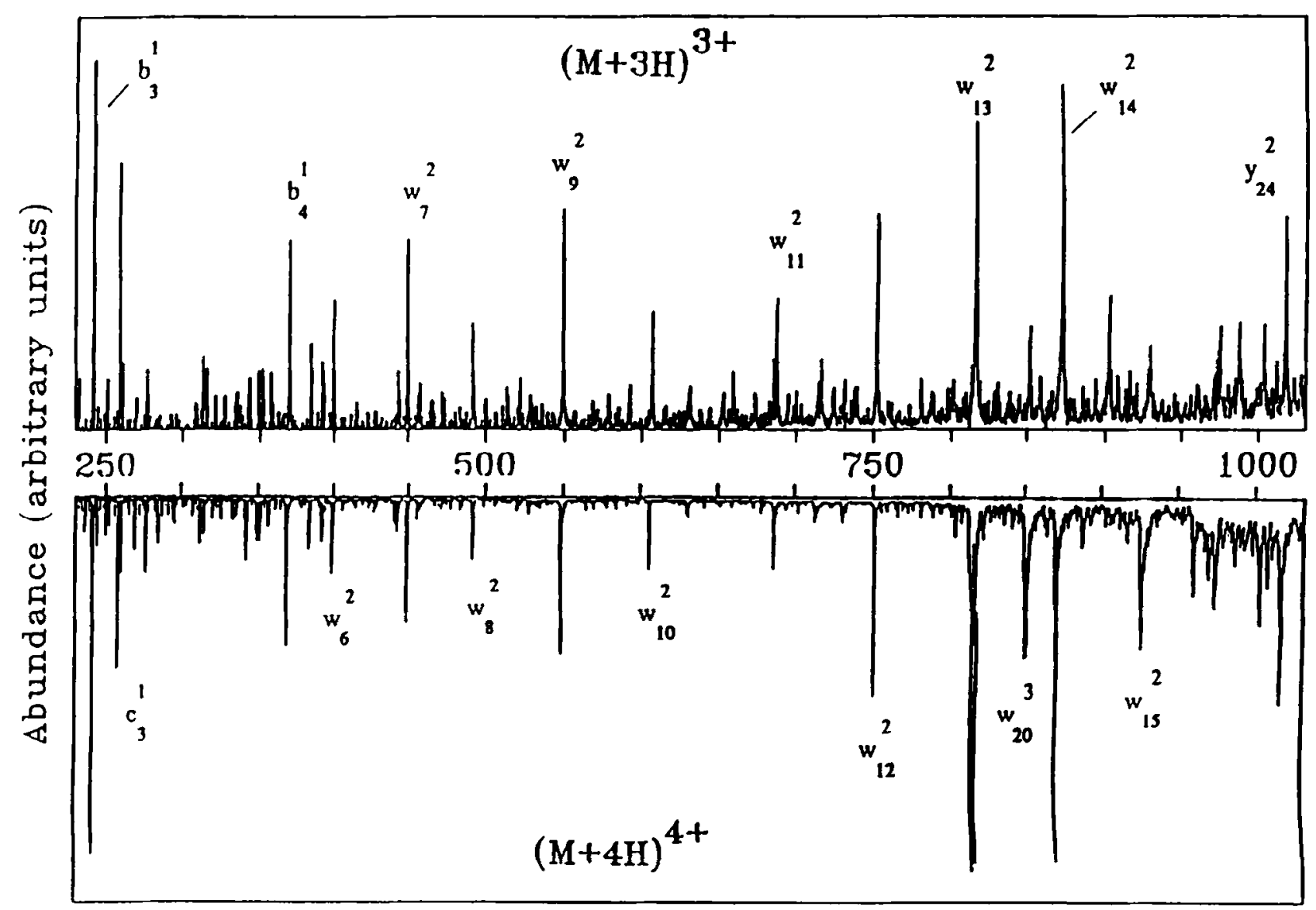

Figure 7. Comparison of the low mass regions from the product ion spectra of the triply and the quadruply protonated molecular ions of pancreatic polypeptide (avian), MW $=4237.6 \mathrm{u}$. 
shown) are also virtually identical. This similarity in the tandem mass spectra from precursor ions in various charge states also was observed with the other polypeptides used in this study. Because the tandem mass spectra of precursors in different charge states provide no additional structural information, we have included only the tandem mass spectrum from the most abundant molecular ion for each polypeptide, with the exception of this example.

Charge state assignments. With all of the peptides used in this study, assignment of the charge state of the fragment ions was relatively easy because their amino acid sequences were known. However, the task would be rather complicated, if not impossible, in the case of an unknown sample of peptides of this size. With this difficulty in mind, the resolving power of MS1 was decreased to approximately 500 so that a range of isotopic variants would be included in the precursor ion and analyzed by CA. In accordance with previous work on both molecular ions [18-21] and fragment ions $[13,14,18]$, the charge state of the fragment ions then can be determined from the spacing of the isotopic peaks, provided that they can be resolved in MS2. As demonstrated by the expanded region of the triply charged $w_{19}$ ions in the tandem mass spectrum of the $[\mathrm{M}+5 \mathrm{H}]^{5+}$ ion of galanin message-associated peptide (Figure 8), the actual resolution attained in MS2 is approximately 3600 full width half maximum (FWHM). We suspect that the isotopic distributions of these two ions do not match those expected for molecules of this mass because of poor ion statistics, that is, an insufficient number of these fragment ions was detected to give the expected isotope distributions. The $m / z 0.32$ spacing between the isotopic peaks clearly identifies these ions as being triply charged. Although this resolution was insufficient to provide a $50 \%$ valley resolution for the isotopic peaks from all of

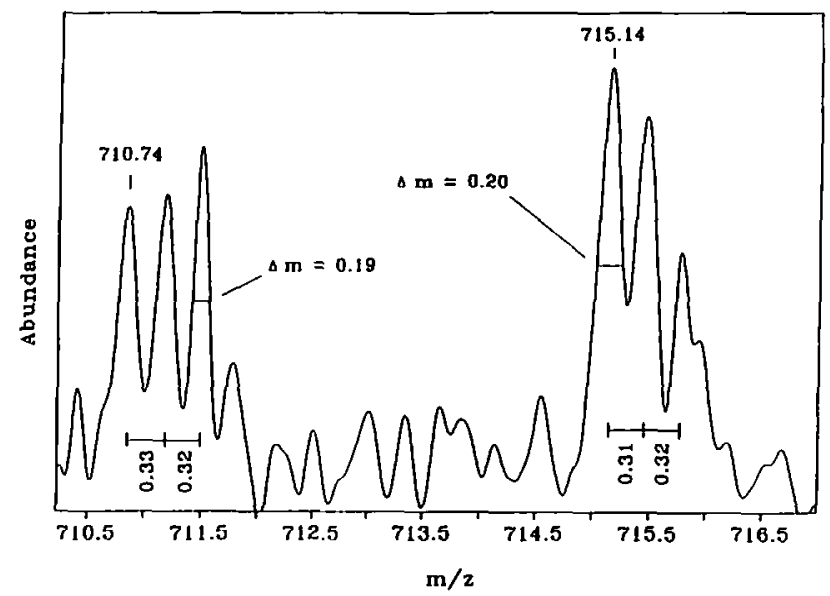

Figure 8. The expanded region of the triply charged $w_{19}$ ions in the product ion spectrum of the quintuply protonated molecular ion $[\mathrm{M}+5 \mathrm{H}]^{5+}$ of galanin message-associated peptide fragment $1-41$ amide (porcine), $\mathrm{MW}=4643.2 \mathrm{u}$. the fragment ions, it allowed the charge states from all the fragment ions to be assigned. The ability to determine the charge states of the fragment ions permits the mass to be calculated for the observed fragment ions. With this information, tandem mass spectra from multiply charged precursors can be interpreted with no more difficulty than interpretation of the tandem mass spectrum from a singly charged peptide. Unfortunately, further increases in the size of the analyte may make this approach impractical on sector instruments due to the limited resolution of array detectors $(\sim 5000$ FWHM) [17]. A potential problem can arise in this approach from overlapping isotopic patterns from different fragments with coincidentally very similar mass-to-charge ratio values.

It also should be pointed out that the mass accuracy of these measurements is very good, as shown by the two $w_{19}^{3}$ ions from galanin message-associated peptide (Figure 8). The experimental values of these ions are within $\pm 0.4 \mathrm{u}$ of their theoretical monoisotopic masses [2128.8 and $2142.6 \mathrm{u}$ (calculated) versus 2129.2 and 2142.4 u (experimental)]. Similar mass accuracies (within $\pm 0.4 \mathrm{u}$ ) were obtained for the other fragments in this spectrum and for the tandem mass spectra of the other peptides discussed. The combination of excellent mass accuracy with the ability to assign charge states is expected to greatly facilitate the interpretation of tandem mass spectra from large unknown peptides.

\section{Conclusions}

The same type of fragmentation observed in the high energy CA spectra of singly charged peptides $[1,21]$ is also seen in the tandem mass spectra of multiply protonated peptides. As demonstrated by this study, high energy CA can provide complete sequence information from peptides in the 4-5-ku range. In addition, these collision energies permit the formation of $d, v$, and $w$ ions to differentiate isomeric amino acids.

Although the peptides used in this study have not been analyzed previously by tandem mass spectrometry, their high energy CA spectra can be compared to those in the literature obtained by low energy collisions of similarly sized peptides [7-10]. In these previous studies, tandem mass spectrometric analysis of multiply charged melittin (2844 u) via low energy CA provided nearly the entire sequence [9]. However, when the size of the peptides was increased to $4 \mathrm{ku}$, large portions of the sequence could not be determined $[7,8]$. For example, only approximately one third of the sequence of human parathyroid hormone (MW = $5064 \mathrm{u}$ ) could be obtained in this manner [7]. By contrast, the high energy collisions used in this study provide information from all regions of the peptides studied with molecular weights ranging from 4181 to 4920 u.

The tandem mass spectra of multiply charged precursor ions produce fragment ions whose charge states range from singly charged to the charge state of the 
precursor. The charge states of the fragment ions correlate well with the number of basic residues (i.e., arginines, lysines, histidines, and the original $\mathrm{N}$ terminus) contained in the fragment ion. The resolutions provided by four-sector mass spectrometers permit the charge state of the fragment ions to be determined by identifying the spacing between the isotopic peaks of the fragment ions. This eliminates the complexity in spectral interpretation caused by the presence of fragment ions in various charge states. The high degree of mass accuracy achieved with this type of instrument permits identification of amino acids that differ by as little as a single unit. The combination of excellent mass accuracy with the ability to assign charge states of the fragment ions is expected to greatly facilitate the interpretation of tandem mass spectra from unknown peptides.

The use of ESI has significantly reduced the amount of material needed for tandem mass spectrometric analysis of large multiply charged peptide ions as compared with FAB. In our previous studies with FAB as the ionization source, $10-100 \mathrm{nmol}$ of a peptide was needed solely to acquire a tandem mass spectrum, that is, additional sample was required to tune the instrument, determine the molecular weight of the peptide, and set up the tandem mass spectrometry experiment [11-13]. The use of ESI in the current study has decreased sample requirements to the level where 500 pmol of a sample is sufficient to tune the mass spectrometer and to acquire both the mass and tandem mass spectra. We are currently researching methods to further reduce sample requirements for tandem mass spectrometric analysis of large multiply charged peptide ions.

The capabilities described here lead us to propose the use of tandem mass spectrometric analysis of multiply charged precursor ions on a tandem four-sector instrument as a strategy to directly obtain the amino acid sequences of peptides up to $5 \mathrm{ku}$ on a routine basis. However, it should be noted that tandem mass spectrometry does not always permit the complete sequence to be determined. This ability is important for peptides that are resistant to chemical and enzymatic digestion, such as the $\beta$-amyloid peptides and the membrane spanning regions of transmembrane proteins. Additionally, the capacity to sequence the intact peptides decreases the amount of sample preparation and losses associated with these procedures.

\section{Acknowledgments}

This work was partially supported by a grant from the U.S. National Institutes of Health (5-P41-RR0535-05). All mass spectra were recorded at the Suntory Institute for Bioorganic Research, Osaka, Japan.

\section{References}

1. Biemann, K.; Scoble, H. A. Science 1987, 237, 992.

2. Matsuda, H.; Takekiyo, M.; Katakuse, I.; Wada, Y. In Mass Spectrometry of Peptides; Desiderio, D. M., Ed.; CRC Press: Boca Raton, 1991; pp 233.

3. Burlingame, A. L.; Baillie, T. A.; Derrick, P. J. Anal. Chem. 1986, 58, 165R.

4. Crabb, J. W.; Johnson, C. M.; Carr, S. A.; Armes, L. G.; Sarri, J. C. J. Biol. Chem. 1988, 263, 18678.

5. Kenny, P. T. M.; Orlando, R. Anal. Chem. 1992, 64, 952.

6. Ashcroft, A. E.; Derrick, P. J. In Mass Spectrometry of Peptides; Desiderio, D. M., Ed.; CRC Press: Boca Raton, 1991; pp 121.

7. Smith, R. D.; Loo, J. A.; Edmonds, C. G.; Barinaga, C. J.; Udseth, H. R. Anal. Chem. 1990, 62, 882.

8. Smith, R. D.; Loo, J. A.; Barinaga, C. J.; Edmonds, C. G.; Udseth, H. R. J. Am. Soc. Mass Spectrom. 1990, 1, 53.

9. Barinaga, C. J.; Edmonds, C. G.; Udseth, H. R.; Smith, R. D. Rapid Commun. Mass Spectrom. 1989, 3, 160.

10. Loo, J. A.; Edmonds, C. G.; Smith, R. D. Anal. Chem. 1991, 63, 2488.

11. Orlando, R.; Fenselau, C. Org. Mass Spectrom. 1991, 26, 664.

12. Orlando, R.; Boyd, R. K. Org. Mass Spectrom. 1992, 27, 151.

13. Orlando, R.; Vassilev, V. P. Biol. Mass Spectrom. 1992, 21, 385.

14. Orlando, R. In Proceedings of the 40th Conference on Mass Spectrometry and Allied Topics; Washington, DC., June 1992.

15. Farbris, D.; Kelly, M.; Murphy, C.; Wu, Z.; Fenselau, C. I. Am. Soc. Mass Spectrom. 1993, 4, 652.

16. Downard, K. M.; Biemann, K. In Proceedings of the $42 n d$ Conference on Mass Spectrometry and Allied Topics; Chicago, IL, May 1994.

17. Hill, J. A.; Biller, J. E.; Biemann, K. Int. J. Mass Spectrom. Ion Processes 1992, 111, 1.

18. Meng, C. K.; McEwen, C. N.; Larsen, B. S. Rapid Commun. Muss Spectrom. 1990, 4, 151.

19. Henry, K. D.; McLafferty, F. W. Org. Mass Spectrom. 1990, 25, 490.

20. Schwartz, J. C.; Syka, J. E. P.; Jardine, I. I. Am. Soc. Mass Spectrom. 1991, 2, 198.

21. Biemann, K. Annu. Rev. Biochemt. 1992, 61, 977. 